\title{
MAPEAMENTO E CRUZAMENTO DAS INFORMAÇÕES SOBRE AVALIAÇÃO CLÍNICA, DIAGNÓSTICO E INTERVENÇÕES DE FISIOTERAPIA ${ }^{1}$
}

\author{
Nayala Lirio Gomes Gazola², Grace Teresinha Marcon Dal Sasso33, Carolina Luana de Mello4, Paulino Ferreira de \\ Sousa ${ }^{5}$
}

${ }^{1}$ Artigo extraído da tese - Registro Eletrônico de Fisioterapia baseado na CIF/2003 para Pacientes de Terapia Intensiva, apresentada ao Programa de Pós-Graduação em Enfermagem (PEN) da Universidade Federal de Santa Catarina (UFSC), em 2015.

${ }^{2}$ Doutora em Enfermagem. Fisioterapeuta do Hospital Universitário Polydoro Ernani de São Thiago (HU/UFSC). Florianópolis, Santa Catarina, Brasil. E-mail: nayalagazola@gmail.com

${ }^{3}$ Doutora em Enfermagem. Professora do PEN/UFSC. Florianópolis, Santa Catarina, Brasil. E-mail: gracetmds@gmail.com

${ }^{4}$ Fisioterapeuta do HU/UFSC. Florianópolis, Santa Catarina, Brasil. E-mail: carol_luana@hotmail.com

${ }^{5}$ Doutor em Enfermagem. Professor do Curso de Pós-Graduação em Sistemas de Informação de Enfermagem da Escola Superior de Enfermagem do Porto. Porto, Portugal. E-mail: paulino@esenf.pt

\section{RESUMO}

Objetivo: descrever a experiência para desenvolver um registro eletrônico em fisioterapia baseado na Classificação Internacional de Funcionalidade, Incapacidade e Saúde através do mapeamento e cruzamento de informações da avaliação clínica, diagnóstico e intervenções fisioterapêuticas.

Método: relato de experiência, organizado em quatro etapas: Aprofundamento teórico da Classificação Internacional de Funcionalidade; Aprofundamento teórico da avaliação em fisioterapia; Estruturação dos dados de avaliação, dos diagnósticos e intervenção fisioterapêutica; Informatização e integração de forma sistematizada dos dados da avaliação clínica, dos diagnósticos e intervenções.

Resultados: a estruturação dos dados foi dividida por sistemas do corpo humano. Sendo assim, para o sistema respiratório construiu-se 15 tópicos de avaliação, 145 diagnósticos e 74 intervenções; para o sistema neuromusculoesquelético construiu-se 37 tópicos de avaliação, 132 diagnósticos e 65 intervenções; para o sistema cardiovascular construiu-se cinco tópicos de avaliação, 23 diagnósticos e cinco intervenções e para o sistema gastrointestinal e renal construiu-se sete tópicos de avaliação, 21 diagnósticos e uma intervenção. Desta forma, foram construídos 64 itens de avaliação, 318 diagnósticos baseados na Classificação de Funcionalidade e 145 intervenções fisioterapêuticas.

Conclusão: o mapeamento das informações sobre avaliação fisioterapêutica, diagnósticos e intervenções para integrar um registro eletrônico em fisioterapia tornará o uso da Classificação Internacional de Funcionalidade, Incapacidade e Saúde mais segura e rápida possibilitando sua aplicabilidade diária na clínica hospitalar.

DESCRITORES: Classificação internacional de funcionalidade, incapacidade e saúde. Informática. Fisioterapia. Cuidados críticos. Registros eletrônicos de saúde.

\section{MAPPING AND CROSS-CHECKING INFORMATION REGARDING CLINCIAL EVALUATION, DIAGNOSES AND PHYSIOTHERAPEUTIC INTERVENTIONS}

\begin{abstract}
Objective: to describe the experience of developing an electronic health record in physiotherapy based on the International Classification of Functioning Disability and Health through the mapping and cross-referencing of clinical evaluation information, diagnosis and physiotherapeutic interventions.

Method: an experience report, organized in four stages: Theoretical development of the International Classification of Functionality; Theoretical development of evaluation in physiotherapy; Structuring of evaluation data, diagnoses and physiotherapeutic intervention; Computerization and systematized integration of data from clinical evaluation, diagnoses and interventions.

Results: the structure of the data was divided by systems of the human body. Thus, for the respiratory system, 15 evaluation topics, 145 diagnoses and 74 interventions were constructed; for the neuromusculoskeletal system, 37 evaluation topics, 132 diagnoses and 65 interventions were constructed; for the cardiovascular system, five evaluation topics, 23 diagnoses and five interventions were constructed; and seven assessment topics, 21 diagnoses and one intervention were constructed for the gastrointestinal and renal systems. Thus, 64 evaluation items were constructed, 318 diagnoses based on the Functionality Classification and 145 physiotherapeutic interventions.

Conclusion: the mapping of information regarding physiotherapeutic evaluation, diagnoses and interventions to integrate an electronic physiotherapy records will make use of the International Classification of Functioning, Disability and Health safer and faster, allowing it to be applied in the hospital setting.

DESCRIPTORS: International classification of functioning, disability and health. informatics. Physiotherapy. Critical care. Eletronic health records.
\end{abstract}




\title{
LEVANTAMIENTO Y CRUZAMIENTO DE LAS INFORMACIONES SOBRE EVALUACIÓN CLÍNICA, DIAGNÓSTICO E INTERVENCIONES DE FISIOTERAPIA
}

\begin{abstract}
RESUMEN
Objetivo: describir la experiencia para desarrollar un registro electrónico en fisioterapia basado en la Clasificación Internacional de Funcionalidad, Incapacidad y Salud a través del levantamiento y cruzamiento de informaciones de la evaluación clínica, diagnóstico e intervenciones fisioterapéuticas.

Método: relato de experiencia realizado en cuatro etapas: Profundización teórica de la Clasificación Internacional de Funcionalidad; Profundización teórica de la evaluación en fisioterapia; Estructuración de los datos de evaluación, diagnósticos e intervención fisioterapéutica; Informatización e integración de forma sistematizada de los datos de la evaluación clínica, diagnósticos e intervenciones.

Resultados: la estructuración de los datos fue dividida por sistemas del cuerpo humano. Así, para el sistema respiratorio se construyeron 15 tópicos de evaluación, 145 diagnósticos y 74 intervenciones. Para el sistema neuromusculoesquelético se construyeron 37 tópicos de evaluación, 132 diagnósticos y 65 intervenciones. Para el sistema cardiovascular se construyeron cinco tópicos de evaluación, 23 diagnósticos y cinco intervenciones y para el sistema gastrointestinal y renal se construyeron siete tópicos de evaluación, 21 diagnósticos y una intervención. De esta forma, se construyeron 64 ítems de evaluación, 318 diagnósticos basados en la Clasificación de Funcionalidad y 145 intervenciones fisioterapéuticas.

Conclusión: el levantamiento de las informaciones sobre la evaluación fisioterapéutica, diagnósticos e intervenciones para integrar un registro electrónico en fisioterapia hará que el uso de la Clasificación Internacional de Funcionalidad, Incapacidad y Salud sea más seguro y rápido, posibilitando su aplicabilidad diaria en la clínica hospitalaria.

DESCRIPTORES: Clasificación internacional de funcionalidad. incapacidade y salud. Informática. Fisioterapia. Cuidados críticos. Registros eletrónicos de salud.
\end{abstract}

\section{INTRODUÇÃO}

A atuação do fisioterapeuta em uma unidade de terapia intensiva (UTI) consiste em manter a funcionalidade do paciente por meio da prevenção e tratamento de alterações osteomioarticulares e de complicações respiratórias, monitorização da mecânica respiratória e de trocas gasosas, gerenciamento da ventilação invasiva e não invasiva, cuidado com a via aérea, desmame e extubação, além de participar da equipe multidisciplinar para melhoria do atendimento ao paciente. ${ }^{1}$

Neste contexto, o processo de cuidado de fisioterapia aos pacientes envolve a avaliação, a identificação das disfunções e das limitações funcionais associados ao problema, a definição de metas centradas no paciente, a seleção da intervenção terapêutica efetiva e a avaliação do efeito da intervenção a partir da evolução do paciente. ${ }^{2}$

Autores ressaltam a importância da sistematização da prática dos profissionais fisioterapeutas, podendo esta ser viabilizada pelo uso de testes padronizados para documentação do progresso do paciente antes, durante e após a intervenção. Contudo, uma prática sistematizada também inclui a elaboração de intervenções a partir da avaliação para atingir um determinado objetivo terapêutico. ${ }^{1-2}$

Na perspectiva da sistematização, cada especialidade deve fazer uso de uma terminologia própria, adequada as suas necessidades e fundamentada na exatidão do significado das palavras, porém cada termo deve ser aceito pela comunidade científica e comum a todas as culturas, com as de- vidas adequações semânticas, para possibilitar o intercâmbio do conhecimento. ${ }^{1}$

Com este propósito, em relação ao diagnóstico funcional, o Conselho Federal de Fisioterapia e Terapia Ocupacional recomenda aos fisioterapeutas o uso da Classificação Internacional de Funcionalidade, Incapacidade e Saúde (CIF) para a padronização, uniformização e utilização de uma linguagem comum. Pertencente ao grupo das classificações internacionais desenvolvida pela Organização Mundial de Saúde a CIF foi elaborada com o propósito de registrar e organizar o imenso volume de informações das condições de saúde e o estado funcional dos indivíduos, bem como a capacidade de interação destes com a vida cotidiana, profissional, familiar e social. ${ }^{3-4}$

O processo de mapeamento e cruzamento dos dados da avaliação do paciente com o diagnóstico baseado na CIF tem com o objetivo traçar intervenções adequadas, eficazes e seguras ao paciente internado na UTI. Contudo, o grande volume de informações torna muitas vezes seu uso limitado na prática devido ao tempo que o profissional teria que dispor para elaborar, organizar e descrever estas informações.

Um registro eletrônico em Saúde (RES) consiste em um sistema para registro, recuperação e manipulação das informações a respeito da saúde dos indivíduos, numa forma processável eletronicamente permitindo a comunicação entre pares promovendo o cuidado eficaz, qualificado e seguro. ${ }^{5-6}$ Devido a este fato, a criação de um sistema de registros tem 
por objetivo contribuir para a sistematização da assistência das profissões, incluindo a fisioterapia, visando o pensamento crítico, a tomada de decisão segura, raciocínio investigativo e a busca constante de informações e evidências científicas, tendo como meta a melhoria da saúde da população. ${ }^{7-8}$

Atualmente, considerando o grande volume de dados e informações advindos da assistência fisioterapêutica, torna-se urgente a necessidade de criação de um sistema informatizado que os gerencie e os disponibilize de forma padronizada, otimizando o tempo do profissional e facilitando o acesso à informação. Além disso, permitir a apresentação dos dados de forma dinâmica e apresentar uma interface amigável ao usuário possibilitando um estudo epidemiológico também é de grande interesse para a sociedade. ${ }^{9}$ Para isto, um registro eletrônico de fisioterapia para UTI deve possuir uma terminologia padrão utilizada pelos fisioterapeutas especialistas nesta área, tanto no que diz respeito à avaliação quanto ao diagnóstico baseado na CIF e nas respectivas intervenções. Assim, para melhorar sua prática clínica, a fisioterapia necessita de sistemas eletrônicos de informações interpretáveis, fáceis de usar e seguros. ${ }^{10}$

Por fim, o desenvolvimento de uma proposta de registro eletrônico em fisioterapia que atenda pacientes internados em UTI tem como finalidade o maior controle, rigor, medidas de qualidade e a continuidade da assistência da fisioterapia. Bem como, fornecer aos profissionais uma ferramenta de suporte para a tomada de decisão segura, garantir a continuidade da intervenção e a autonomia para estabelecer seus diagnósticos.

Este manuscrito descreve a experiência da articulação realizada para desenvolver o registro eletrônico em fisioterapia baseado na Classificação Internacional de Funcionalidade, Incapacidade e Saúde através do mapeamento e cruzamento de informações a respeito da avaliação clínica, diagnóstico e intervenções fisioterapêuticas.

\section{MÉTODO}

O presente relato de experiência que descreve a estruturação do registro eletrônico em fisioterapia baseado na CIF/2003, realizado nas dependências do Grupo de Pesquisa Clínica, Tecnologias e Informática em Saúde e Enfermagem do Programa de Pós-Graduação em Enfermagem da Universidade Federal de Santa Catarina

Participaram da realização do mapeamento e das informações clínicas duas fisioterapeutas, uma doutora, ambas especialistas em terapia intensiva e com experiência de mais de 6 anos nesta área, e uma professora doutora do Departamento de Enfermagem da UFSC. O mapeamento e o cruzamento das avaliações clínicas, dos diagnósticos baseado na CIF e das intervenções fisioterapêuticas foi construído de março de 2013 a junho de 2014 e seguiu as seguintes etapas (Figura 1).

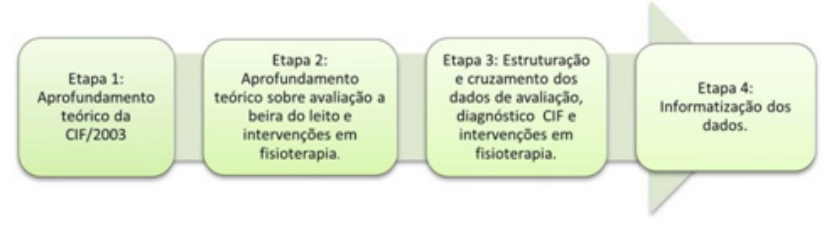

Figura 1 - Fluxograma das etapas do estudo

Primeira etapa: Aprofundamento teórico da CIF/2003. Esta etapa foi construída a partir da leitura e compreensão da CIF, bem como do levantamento dos estudos em bases de dados sobre a utilização da CIF no ambiente hospitalar, em especial em terapia intensiva, desde a sua evolução histórica até a aplicabilidade clínica.

Segunda etapa: Aprofundamento teórico da avaliação em fisioterapia à beira do leito realizada em livros e artigos científicos. Aprofundamento teórico das intervenção fisioterapêutica de acordo com as Recomendações de Fisioterapia para o Paciente Adulto Crítico e com o I Consenso de Termos em Fisioterapia. ${ }^{11-12}$ Após este processo, os dados foram divididos didaticamente em sistemas, sendo: Sistema Respiratório, Sistema Neuromusculoesquelético, Sistema Cardiovascular e Sistema Gastrointestinal e Renal. Além de uma estrutura livre para anotações de dados da Ventilação Mecânica Invasiva e outra para Ventilação Mecânica Não Invasiva. Esta etapa foi desenvolvida no programa Word ${ }^{\circledR}$.

Terceira etapa: Estruturação dos dados de avaliação, classificação dos diagnósticos de acordo com a CIF e intervenção fisioterapêtica em planilha eletrônica do programa Excel ${ }^{\circledR}$. Nesta etapa, duas fisioterapeutas com experiência em fisioterapia hospitalar e terapia intensiva realizaram a articulação dos dados de avaliação e intervenção partindo do diagnóstico baseado na CIF. ${ }^{3}$ Desta forma, para cada diagnóstico da CIF houve pelo menos um dado de avaliação e um dado de intervenção fisioterapêutica. Vale ressaltar, que nem todos os dados da avaliação e do diagnóstico CIF tinham uma intervenção direta da fisioterapia, pois, em muitas situações clínicas o fisioterapeuta necessita avaliar e nem sempre executa uma intervenção ou mesmo contra indica a fisioterapia. 
Quarta etapa: Informatização dos dados e construção do sistema informatizado de fisioterapia em terapia intensiva. Esta etapa ocorreu após finalização das anteriores, no mês de outubro de 2014, os dados associados foram informatizados e integraram um sistema que possibilitou organizar de forma sistematizada a avaliação clínica dos pacientes, os diagnósticos da CIF e as intervenções. Neste sistema, o fisioterapeuta terá a possibilidade de registrar a avaliação clínica de cada sistema humano proposto e controlar a ventilação mecânica não invasiva e invasiva. A partir destas informações, o sistema fornece os respectivos diagnósticos da CIF e as sugestões de intervenção fisioterapêutica. Esta etapa foi realizada em conjunto com um programador de sistemas, com experiência em programação para a área da saúde.

\section{RESULTADO DA EXPERIÊNCIA}

O Quadro 1 demonstra as possibilidades de raciocínio clínico que poderão ser utilizadas na prática profissional e parte do resultado oriundo do caminho metodológico percorrido que resultou na articulação dos dados da avaliação, diagnóstico e intervenção fisioterapêutica.

Quadro 1 - Exemplificação da associação dos dados da avaliação clínica, diagnósticos e intervenções de Fisioterapia para o Sistema Respiratório

\begin{tabular}{|c|c|c|}
\hline Avaliação & Diagnóstico CIF/2003* & Intervenção \\
\hline Padrão Ventilatório & $\begin{array}{l}\text { b4408 Funções da Respiração, outras } \\
\text { especificadas }\end{array}$ & \\
\hline $\begin{array}{l}\text { Padrão Ventilatório Diafragmáti- } \\
\text { co/Abdominal }\end{array}$ & $\begin{array}{l}\text { b4408.0 Nenhuma Deficiência nas } \\
\text { Funções da Respiração, outras espe- } \\
\text { cificadas }\end{array}$ & $\begin{array}{l}\text { Posicionamento em Fowler } 45^{\circ} \\
\text { Decúbito dorsal } \\
\text { Inspiração profunda } \\
\text { Inspirometria de incentivo a fluxo } \\
\text { Inspirometria de incentivo a volume }\end{array}$ \\
\hline Padrão Ventilatório Misto & $\begin{array}{l}\text { b4408.1 Deficiência leve nas Funções } \\
\text { da Respiração, outras especificadas }\end{array}$ & $\begin{array}{l}\text { Posicionamento em Fowler } 45^{\circ} \\
\text { Decúbito dorsal } \\
\text { Inspiração profunda } \\
\text { Inspirometria de incentivo a fluxo } \\
\text { Inspirometria de incentivo a volume }\end{array}$ \\
\hline Padrão Ventilatório Apical & $\begin{array}{l}\text { b4408.2 Deficiência moderada nas } \\
\text { Funções da Respiração, outras espe- } \\
\text { cificadas }\end{array}$ & $\begin{array}{l}\text { Posicionamento em Fowler } 45^{\circ} \\
\text { Decúbito dorsal } \\
\text { Inspiração profunda }\end{array}$ \\
\hline $\begin{array}{l}\text { Padrão Ventilatório Paradoxal/ } \\
\text { Invertido }\end{array}$ & $\begin{array}{l}\text { b4408.3 Deficiência grave nas Funções } \\
\text { da Respiração, outras especificadas }\end{array}$ & $\begin{array}{l}\text { Posicionamento em Fowler } 45^{\circ} \\
\text { Decúbito dorsal } \\
\text { Ventilação não invasiva } \\
\text { Ventilação mecânica invasiva } \\
\end{array}$ \\
\hline Sinal de Hoover & $\begin{array}{l}\text { b4408.4 Deficiência completa nas } \\
\text { Funções da Respiração, outras espe- } \\
\text { cificadas }\end{array}$ & $\begin{array}{l}\text { Posicionamento em Fowler } 45^{\circ} \\
\text { Decúbito dorsal } \\
\text { Ventilação não invasiva } \\
\text { Ventilação mecânica invasiva }\end{array}$ \\
\hline Ventilação Mecânica & b4408.9 Não aplicável & Posicionamento em Fowler $45^{\circ}$ \\
\hline
\end{tabular}

*Classificação Internacional de Funcionalidade, Incapacidade e Saúde.

Foram construídos um total de 64 itens de avaliação à beira do leito para pacientes internados na UTI (Quadro 2). A construção foi dividida por sistemas humanos com o objetivo de seguir os moldes da CIF e por ser didático, a fim de estabelecer uma padronização e facilitar a utilização diária da evolução do atendimento fisioterapêutico realizado, assim divididos: Sistema Respiratório (15 tópicos); Sistema Neuromusculoesquelético (37 tópicos); Sistema Cardiovascular (sete tópicos); Sistema Gastrointestinal e Renal (sete tópicos). Das associações com a CIF/2003 surgiram 318 diagnós- ticos, sendo assim divididas: Sistema Respiratório (142 diagnósticos); Sistema Neuromusculoesquelético (132 diagnósticos); Sistema Cardiovascular (23 diagnósticos); Sistema Gastrointestinal e Renal (21 diagnósticos). Por fim, após a realização do levantamento dos critérios da avaliação clínica, para cada item e subitem foi construído um diagnóstico da CIF e, posteriormente, foi estruturada uma lista de possíveis intervenções que o fisioterapeuta poderá selecionar como julgar pertinente para cada paciente em determinada situação clínica. Desta forma, foram construídas 145 possibilidades de in- 
tervenção, assim divididas: Sistema Respiratório (74 possibilidades de intervenção); Sistema Neuromusculoesquelético (65 possibilidades de intervenção);
Sistema Cardiovascular (cinco possibilidades de intervenção); Sistema Gastrointestinal e Renal (uma possibilidade de intervenção).

Quadro 2 - Resultado da associação da avaliação, diagnósticos baseados na CIF/2003 e intervenções de fisioterapia para terapia intensiva. Florianópolis, SC, Brasil, 2014

\begin{tabular}{lccc}
\hline & Avaliações & $\begin{array}{c}\text { Diagnósticos } \\
\text { CIF/2003* }\end{array}$ & Intervenções \\
\hline Sistema Respiratório & 15 & 142 & 74 \\
Sistema Neuromusculoesquelético & 37 & 132 & 65 \\
Sistema Cardiovascular & 5 & 23 & 05 \\
Sistema Gastrointenstinal e Renal & 7 & 21 & 01 \\
Total & 64 & 318 & 145 \\
\hline
\end{tabular}

*Classificação Internacional de Funcionalidade, Incapacidade e Saúde

Primeiramente, a construção da avaliação fisioterapêutica foi dinâmica e por meio de uma revisão extensa da literatura, buscaram-se informações necessárias para subsidiar o diagnóstico e conduzir o processo de tomada de decisão para a conduta fisioterapêutica. ${ }^{13}$

O Quadro 1 tem por objetivo demonstrar uma pequena parte da construção das associações de avaliação, diagnóstico CIF e intervenções. Desta forma, a coluna 1 é composta por dados da avaliação, sendo dividida em sistemas: avaliação do sistema respiratório (saturação periférica de oxigênio, expansibilidade torácica, padrão respiratório, sinais e sintomas de desconforto respiratório, palpação, ausculta pulmonar); a avaliação do sistema neuromusculoesquelético (nível de consciência pela escala de coma de Glasgow, escala de sedação de Ramsay e a escala de agitação e sedação de Richmond (RASS, do inglês Richmond Agitation-Sedation Scale), tônus muscular, déficit motor, restrição articular, dor, força muscular); avaliação do sistema cardiovascular (sinais vitais, uso de drogas vasoativas) e avaliação do sistema gastrointestinal e renal (náusea, vômito, melena, diurese) além de abordar os exames complementares como exames de imagem (raio-X), medidas de variáveis respiratórias (força muscular) e gasometria arterial.

Posteriormente, para a construção do diagnóstico funcional, optou-se pelo uso da CIF atendendo as recomendações do Conselho Nacional de Fisioterapia e Terapia Ocupacional. ${ }^{14}$ A CIF dividi-se em duas partes: A primeira parte trata dos conceitos de Funcionalidade e Incapacidade compostos pelos componentes: Funções e Estruturas do corpo; e Atividades e participação. Os componentes do Corpo contêm oito capítulos para as funções dos sistemas orgânicos e oito capítulos para as estrutu- ras do corpo. Os componentes da Funcionalidade contêm nove capítulos para as atividades e para a participação. Os capítulos das atividades e da participação vão do contato do sujeito consigo mesmo à sua inserção político-social na comunidade, do ambiente mais próximo do sujeito, mais imediato para o indivíduo, até o ambiente social. Ressalta-se que cada capítulo é composto por subcategorias mais detalhadas. A segunda parte aborda os Fatores contextuais compostos dois componentes: Fatores ambientais e Fatores pessoais. Os Fatores Ambientais contêm cinco capítulos que causam impacto positivo ou negativo, na relação do sujeito com o ambiente humano, físico, social, político e ambiental. Quanto aos Fatores Pessoais, não são classificados pela CIF devido à grande variação social e cultural que estão envolvidas com as diferentes pessoas no mundo. . $^{3,15-16}$

A coluna 2 do Quadro 1, por sua vez, demonstra os diagnósticos elaborados a partir da CIF e as partes que foram utilizadas neste estudo. Em relação às funções e estruturas do corpo foram selecionadas: funções do sistema respiratório (b440-b449), funções mentais (b110-b114), funções do sistema cardiovascular (b410-439), funções do sistema neuromusculoesquelético (b710-b789), funções do sistema gastrointestinal e renal (b510-b539, b610-b639). Estrutura do sistema respiratório (s430), a estrutura do sistema neuromusculoesquelético (s710-s799). Em relação aos Fatores ambientais somente foram incluídos os produtos e tecnologias (e1101, e198).

A interpretação destes diagnósticos surgiu a partir de uma análise criteriosa do processo de avaliação que tem por objetivo identificar o fator do comprometimento funcional para elaborar a intervenção fisioterapêutica mais adequada. ${ }^{13,17}$ Neste estudo, optou-se por utilizar apenas estas partes, pois 
atendiam ao objetivo do trabalho que foi construir uma ferramenta de avaliação, diagnóstico e intervenção, especificamente, para pacientes internados em UTI. Em conformidade com estes achados, um estudo que teve por objetivo identificar as categorias da CIF mais utilizadas na prática do fisioterapeuta, demonstrou que as funções sensórias foram utilizadas em 1752 casos, função cardiovascular e respiratória em 1064 casos e a função neuromusculoesquelética em 3485 casos, bem como, as estruturas do sistema nervoso em 359 casos, estruturas do sistema cardiovascular e respiratório em 5158 casos e as estruturas do sistema neuromusculoesqueléticas e do movimento em 5158 casos. $^{18}$

Por fim, a elaboração das intervenções fisioterapêuticas teve por finalidade orientar e não impor uma conduta a ser utilizada. A construção destas intervenções foi pautada em estudos com diversos níveis de recomendação publicados nos últimos anos. ${ }^{11,19-29}$

Ainda, a coluna 3 do Quadro 1 demonstra as técnicas e recursos de fisioterapia que visam aprimorar a funcionalidade e reduzir as incapacidades respiratórias e motoras relacionadas à internação em UTI. Estes recursos visam a remoção de secreções, ${ }^{23-24}$ a expansão pulmonar, ${ }^{25}$ o treinamento da musculatura respiratória, ${ }^{26}$ a mobilização precoce ${ }^{27}$ e o posicionamento dos pacientes criticamente doentes. ${ }^{11,21,28-29}$

Quanto as avaliações que não receberam intervenção, cita-se como exemplo a avaliação do fisioterapeuta da presença de melena, o diagnóstico da CIF é b5258.3 (Deficiência grave nas funções de defecação, outras especificadas), porém nesta situação não há uma atuação da fisioterapia específica para melena, o profissional avalia mas não atua.

Desta forma, a assistência fisioterapêutica no cuidado do paciente internado em UTI deve identificar precocemente os problemas cinético-funcionais e um programa de reabilitação é recomendado como crucial e seguro para recuperação destes pacientes. ${ }^{30-31}$

A limitação do estudo consistiu na dificuldade em associar todos os itens de avaliação do paciente grave utilizados pelo fisioterapeuta na UTI com os diagnósticos da CIF.

\section{CONCLUSÃO}

Conclui-se que o mapeamento das informações sobre avaliação fisioterapêutica à beira do leito, diagnósticos e intervenções visam desenvolver uma estrutura de dados para integrar um registro eletrônico em fisioterapia. Este tem potencial para tornar o uso da CIF mais segura e rápida, possibilitando sua aplicabilidade diária na clínica hospitalar, pois se sabe que, mesmo com as versões resumidas (core sets) sua utilização é limitada na prática. Este estudo visou contribuir para a evolução e aplicação da CIF, que traça como planos futuros, o desenvolvimento de instrumentos de avaliação que permitem identificar e medir a condição clínica do paciente, o desenvolvimento de investigações sobre tratamentos e intervenções combinadas, bem como proporcionar sua aplicabilidade prática mediante o desenvolvimento e utilização de tecnologias de informática e criação de formulários de registros de casos clínicos.

Além disso, a sistematização da informação obtida pelo cruzamento destas informações traz inúmeras vantagens, tais como a padronização de termos, o raciocínio clínico e a tomada de decisão segura à beira do leito este processo poderá refletir na melhoria da qualidade da assistência fisioterapêutica aos pacientes graves internados em UTI's.

Em um futuro próximo o armazenamento destas informações de forma sistematizada e padronizada poderão fornecer embasamento para produções científicas, traçar metas para melhorar a qualidade do serviço e medir a evolução dos pacientes, pois fornecerão dados confiáveis e passíveis de comparação.

Para novas pesquisas sugere-se a associação dos diagnósticos da CIF com outros itens de avaliação nas diferentes aéreas do conhecimento da fisioterapia (como por exemplo, ortopedia e neurologia) e com isso a possibilidade do desenvolvimento de registros eletrônicos nestas áreas.

\section{REFERÊNCIAS}

1. Menezes S. Intensive Care Physical Therapy: a new name for an old specialty. ASSOBRAFIR Ciência [Internet]. 2011 [cited 2017 Sep 10]; 2(2):49-53. Available from: http://www.uel.br/revistas/uel/ index.php/rebrafis/article/viewFile/10602/9308

2. Sampaio RF, Mancini MC, Fonseca ST. Produção científica e atuação profissional: aspectos que limitam essa integração na fisioterapia e na terapia ocupacional. Rev Bras Fisioter [Internet]. 2012 [cited 2017 Sep 10]; 6(3):113-8. Available from: http://www.ufjf.br/ especializacaofisioto/files/2010/03/Producaocientifica-e-atuacao-profissional-RBF-20021.pdf

3. Classificação Internacional de Funcionalidade, Incapacidade e Saúde - CIF, 2004 [Internet] [cited 2017 Sep 10]. Available from: http://www.inr.pt/ uploads/docs/cif/CIF_port_\%202004.pdf

4. Bernardes JM, Pereira AA. The International Classification of Functioning, Disability and Health 
(ICF) and its contributions to physical therapy. Fisioter Bras. 2011; 12(1): 58-64.

5. Silva ML, Virginio Junior LA. Sociedade Brasileira de Informática em Saúde - SBIS. Manual de certificação para sistemas de registro eletrônico em saúde (S-RES). Versão 4.2 [Internet] 2016 [cited 2017 Sep 10]. Available from: http://www.sbis.org.br/certificacao/Manual_ Certificacao_SBIS-CFM_2013_v4-1.pdf

6. Barra DCC, Sasso GMT. Data standards, terminology and classification systems for caring in health and nursing. Rev Bras Enferm [Internet]. 2011 [cited 2017 Sep 10]; 64(6): 1141-9. Available from: http:/ / www. scielo.br/pdf/reben/v64n6/v64n6a23.pdf

7. Sasso GTM, Barra DCC, Paese F, Almeida SRW, Rios GC, Marinho MM, Debétio MG. Computerized nursing process: methodology to establish associations between clinical assessment, diagnosis, interventions, and outcomes. Rer Esc Enferm USP [Internet]. 2013 [cited 2017 Sep 10]; 47(1):242-9. Available from: http:/ / www.scielo.br/pdf/reeusp/v47n1/en_a31v47n1.pdf

8. Barra DCC, Sasso GTM, Almeida SRW, Paese F, Rios GC. Method for the modeling and structuring of computerized nursing in intensive care. Texto Contexto Enferm [Internet]. 2016 [cited 2017 Sep 11]; 25 (3): e2380015. Available from: http:/ / www.scielo. br/pdf/tce/v25n3/0104-0707-tce-25-03-2380015.pdf

9. Maggi LE, Rocha PVS, Barbosa PMR, Oliveira RA. Physical therapy record to evaluate amputee patients. Rev Movimenta [Internet]. 2010 [cited 2017 Sep 12]; 3(1):4-15. Available from: http://docplayer.com. br/17962262-Prontuario-eletronico-para-avaliacaofisioterapeutica-de-amputados.html

10. Vreeman DJ, Richoz C. Possibilities and implications of using the ICF and other vocalurary Standards em electronic health records. Physiother Rev Int [Internet] 2013 [cited 2017 Sep 11]; Special Issue Paper. Available from: https://www.ncbi.nlm.nih.gov/pmc/articles/ PMC3907616/pdf/nihms517899.pdf

11. França EET, Ferrari F, Fernandes P, Cavalcanti R, Duarte A, Matinez BP, et al. Physical therapy in critically ill adult patients: recommendations from the Brazilian Association of Intensive Care Medicine Department of Physical Therapy. Rev Bras Ter Intensiva [Internet]. 2012 [cited 2017 Sep 11]; 24(1):622. Available from: http://www.scielo.br/pdf/rbti/ v24n1/en_03.pdf

12. Costa RP, Castro AA, Feltrim MIZ, Neiva PD, Menezes SLS, organizadores. I Consenso de termos em fisioterapia respiratória. Associação de diretrizes e terminologia em fisioterapia respiratória e terapia intensiva. ASSOBRAFIR [Internet] [cited 2017 Sep 11]. Available from: http://professor.pucgoias.edu. br/SiteDocente/admin/arquivosUpload/12506/ material/terminologia.pdf

13. Ferreira FMM, Macedo JM, Ferreira MMM. Indicadores na área de fisioterapia no ambiente hospitalar; processos e resultados. Programa de Atualização
PROFISIO. Fisioterapia em Terapia Intensiva Adulto. Ciclo 1. Módulo 3. Editora Artmed: Porto Alegre, 2011.

14. Conselho Federal de Fisioterpia e Terapia Ocupacional - COFFITO [Internet] [cited 2017 Sep 11]. Available from: http://www.crefito10.org.br

15. Nunes CMP. Apostila do Curso de Capacitação na CIF. Módulo III. UDESC; 2012.

16. Araújo ES. Apostila do Curso de Capacitação na Classificação Internacional de Funcionalidade, Incapacidade e Saúde - CIF. Módulo IV. UDESC, 2012.

17. Seguel FAG, Bravo AAA, Goic JEL, Ubiergo SU. Feasibility and clinical utility of icf framework in critical ill patients: case report. Ann Musc Disord [Internet]. 2017 [cited 2017 Sep 11]; 1(1):1-7. Available from: https://www. jscimedcentral.com/MusculoskeletalDisorders / musculoskeletaldisorders-1-1002.pdf

18. Mitchell L. Can the International Classification of Functioning, Disability and Health (ICF) provide highlevel descriptions of Scottish physiotherapy cases? Adv Physiother [Internet]. 2008 [cited 2017 Sep 11]; 10:119-26. Available from: http:/ / www.tandfonline. com/doi/full/10.1080/14038190802180204?scroll= top\&needAccess $=$ true

19. Pinheiro AR, Christofoletti G. Motor physical therapy in hospitalized patients in an intensive care unit: a systematic review. Rer Bras Ter intensiva [Internet]. 2012 [cited 2017 Sep 11]; 24(2):188-96. Available from: http:/ / www.scielo.br/pdf/rbti/v24n2/en_16.pdf

20. Morris PE, Griffin L, Berry M, Thompson C, Hite RD, Winklman $C$, et al. Receiving early mobility during na intensive care unit admission is a predictor IF improved outcomes in acute respiratory faliure. Am J Med Sci [Internet]. 2011 [cited 2017 Sep 11]; 341(5): 373-7. Available from: https://www.ncbi.nlm.nih. gov/pmc/articles/PMC3082620/pdf/nihms-265901. pdf

21. Gosselink R, Bott J, Johnson M, Dean E, Nava S, Norrenberg M, et al. Physiotherapy for adult patients with critical illness: recommendations of the European Respiratory Society and European Society of Intensive Care Medicine Task Force on Physiotherapy for Critically Ill Patients. Intensive Care Med [Internet]. 2008 [cited 2017 Sep 11]; Jul; 34(7):1188-99. Available from: http:/ / pt.or.th/download/cpg/C03.pdf

22. Gonçalves M, Winck J. Exploring the potential of mechanical insufflation-exsufflation. Breathe [Internet]. 2008 [cited 2017 Sep 11]; 4(4):326-9. Available from: http://breathe.ersjournals.com/ content/breathe/4/4/326.full.pdf

23. Santos FRA, Scheneider LC, Forgiarini LA, Veronezi J. Effects of manual rib-cage compression versus PEEPZEEP maneuver on respiratory system compliance and oxygenation in patients receiving mechanical ventilation. Rev Bras Ter Intensiva [Internet]. 2009 [cited 2017 Sep 12]; 21(2):155-61. Available from: http:/ / www.scielo.br/pdf/rbti/v21n2/en_07.pdf 
24. Dias CM, Siqueira TM, Faccio TR, Gontijo LC, Salge JASB, Volpe MS, et al. Bronchial hygiene technique with manual hyperinflation and thoracic compression: effectiveness and safety. Rev Bras Ter Intensiva [Internet]. 2011 [cited 2017 Sep 12]; 23(2):190-8. Available from: http://www.scielo.br/pdf/rbti/ v23n2/a12v23n2.pdf

25. Barbas CSV. Brazilian recommendations of mechanical ventilation 2013. Part 2. Rev Bras Ter Intensiva [Internet]. 2014 [cited 2017 Sep 12]; 26(3):215-39. Available from: http://www.scielo.br/pdf/rbti/ v26n3/en_0103-507X-rbti-26-03-0215.pdf

26. Dantas CM, Silva FS, Siqueira HT, Pinto RMF, Matias $S$, Maciel C, et al. Influence of early mobilization on respiratory and peripheral muscle strength in critically ill patients. Rev Bras Ter Intensiva [Internet]. 2012 [cited 2017 Sep 12]; 24(2):173-8. Available from: http://www.scielo.br/pdf/rbti/v24n2/en_13.pdf.

27. Stiller K. Physiotherapy in Intensive Care: An Updated Systematic Review. Chest [Internet]. 2013 [cited 2017 Sep 12]; 144(3):825-47. Available from: http:/ / www.sciencedirect.com/science/article/pii/ S001236921360598X

28. Morris PE, Goad A, Thompson C, Taylor K, Harry B, Passmore L, et al. Early physical care unit mobility therapy in the treatment of acute respiratory failure. Crit Care Med [Internet]. 2008 [cited 2017 Sep 12]; 36(8):2238-43. Available from: http://www.socati. org.br/aulas/fisioterapia_em_uti.pdf

29. Castro AAM, Calil SR, Freitas AS, Oliveira AB, Porto EF. Chest physiotherapy effectiveness to reduce hospitalization and mechanical ventilation length of stay, pulmonary infection rate and mortality in ICU patients. Resp Med [Internet]. 2013 [cited 2017 Sep 12]; 107: 68-74. Available from: http://www. resmedjournal.com/article/S0954-6111(12)00357-5/ pdf

30. Soares TR, Avena KM, Olivieri FM, Feijó LF, Mendes KMB, Souza Filho AS, et al. Withdrawal of bed following mechanic ventilation discontinuation: are there reflexes on mortality and intensive care unit length of stay? Rev Bras Ter Intensiva [Internet]. 2010 [cited 2017Sep 12]; 22(1):27-32. Available from: http:/ / www.scielo.br/pdf/rbti/v22n1/en_a06v22n1.pdf

31. Gosselink R, Clerckx B, Robbeets C, Vanhullebusch T, Vanpee G, Segers J. Physiotherapy in the Intensive Care Unit. Neth J Critical Care [Internet]. 2011 [cited 2017 Sep 12]; 12(2):68-75. Available from: http:// njcc.nl/sites/default/files/NJCC\%2002\%20reviewGosselink.pdf
Correspondência: Nayala Lirio Gomes Gazola

Rua Osni João Vieira, 620, apto 1004

88101-270 - Campinas. São José, SC, Brasil

E-mail: nayalagazola@gmail.com
Recebido: 26 de dezembro de 2016

Aprovado: 26 de setembro de 2017

This is an Open Access article distributed under the terms of the Creative Commons (CC BY). 